\title{
GREAT: GRaphlet Edge-based network AlignmenT
}

\author{
Joseph Crawford and Tijana Milenković ${ }^{*}$ \\ Department of Computer Science and Engineering, Interdisciplinary Center for \\ Network Science and Applications, and ECK Institute for Global Health \\ University of Notre Dame \\ *Corresponding Author (E-mail: tmilenko@nd.edu)
}

\begin{abstract}
Network alignment aims to find regions of topological or functional similarities between networks. In computational biology, it can be used to transfer biological knowledge from a wellstudied species to a poorly-studied species between aligned network regions. Typically, existing network aligners first compute similarities between nodes in different networks (via a node cost function) and then aim to find a high-scoring alignment (node mapping between the networks) with respect to "node conservation", typically the total node cost function over all aligned nodes. Only after an alignment is constructed, the existing methods evaluate its quality with respect to an alternative measure, such as "edge conservation". Thus, we recently aimed to directly optimize edge conservation while constructing an alignment, which improved alignment quality. Here, we approach a novel idea of maximizing both node and edge conservation, and we also approach this idea from a novel perspective, by aligning optimally edges between networks first in order to improve node cost function needed to then align well nodes between the networks. In the process, unlike the existing measures of edge conservation that treat each conserved edge the same, we favor conserved edges that are topologically similar over conserved edges that are topologically dissimilar. We show that our novel method, which we call GRaphlet Edge AlignmenT (GREAT), improves upon state-of-the-art methods that aim to optimize node conservation only or edge conservation only.
\end{abstract}

\section{Background, motivation, and our contribution}

The goal of network (or graph) alignment in computational biology is to find regions of topological or functional similarities between networks of different species. (We note, however, that network alignment has applications in many domains [1, 2,.) The more biological network data is becoming available, the more importance the problem of network alignment gains. This is because network alignment can be used, for example, to transfer functional (e.g., aging-related [3, 4, 5]) knowledge from well annotated species to poorly studied ones between aligned network regions.

There are two categories of network alignment methods: local network alignment (LNA) and global network alignment (GNA). LNA focuses on optimizing similarity between local (small) regions of different networks, plus, it allows for a region in one network to be mapped to multiple regions in another network. This way, LNA is generally unable to find large conserved subgraphs between networks, and also, it can lead to many-to-many node mappings between the networks [6, 7, 8, 9, 10, 11, 12, 13, 14, which might be motivated biologically, but such alignments are hard to characterize in terms of topological alignment quality [15, 16. On the other hand, GNA aims to optimize global (overall) similarity between different networks, and in general (although some exceptions exist [17]), it results in one-to-one (i.e., injective) node mapping [2, 3, 4, 15, 16, 17, 18, 19, 20, 21, 22, 23, 24, 25, 26, 27, 28, 29]. As such, GNA is able to find large conserved subgraphs, and it also allows for quantifying both topological and biological quality of 
the resulting alignments. In this study, we focus on one-to-one GNA due to its recent popularity (and henceforth, we refer to GNA simply as network alignment), but all concepts introduced here can be applied to LNA as well.

We more formally define network alignment as an injective mapping between the nodes of two networks that aligns the networks well with respect to a desired criterion. Network alignment is a computationally hard problem to solve due to the underlying subgraph isomorphism problem, which is NP-complete [30]. The subgraph isomorphism problem aims to find out whether some graph $G_{2}$ contains another graph $G_{1}$ as its exact subgraph. With this in mind, the network alignment problem aims to "fit well" $G_{1}$ into $G_{2}$ when $G_{1}$ is not necessarily an exact subgraph of $G_{2}$. Thus, since network alignment is computationally intractable, all existing algorithms aiming to solve this problem are heuristics.

In general (although there are some exceptions [28]), existing network alignment methods typically contain two algorithmic components: 1) a node cost function and 2) an alignment strategy 3. 4, 15, 16, 17, 18, 22, 23, 24, 31, 32. A node cost function finds pairwise topological (potentially also biological, e.g., protein sequence) similarities (or equivalently, costs) between two nodes from different networks, while the alignment strategy uses these costs to select a high-scoring alignment (out of all possible alignments) typically with respect to the total node cost function over all aligned nodes [28]. Then, the quality of the resulting alignment is evaluated with respect to some other topological measure, which is different than the node cost function that is used to produce the alignment in the first place. (Alignment quality is also measured via a biological measure, such as functional enrichment of aligned node pairs [28.) Typically, one measures the amount of conserved edges, and multiple measures have been proposed for this purpose, with our recent symmetric substructure score $\left(\mathrm{S}^{3}\right)$ being a superior measure [28]. That is, the goal of existing methods is to align nodes well in hope that they will align edges well, but only after the alignment is produced. Hence, recently, we introduced a novel algorithm, called MAGNA, which is capable of optimizing edge conservation directly while an alignment is being constructed [28].

Here, we approach a novel idea of maximizing both node and edge conservation, and we also approach this idea from a novel perspective, by aligning edges between networks in order to improve node cost function. These are the two major novelties of our study that distinguish us from the existing work. In the process, unlike the existing measures of edge conservation that treat each conserved edge the same, we propose a new measure of edge conservation to favor conserved edges that are topologically similar over conserved edges that are topologically dissimilar. This is another of our novelties. We note that a method exists that infers plausibly alignable interactions across protein-protein interaction (PPI) networks of different species [25]. However, this method is guided biologically rather than topologically: it aligns PPIs relying on conservation of their constituent domain interactions, and thus, it aims to address not the problem of subgraph isomorphism nor edge conservation but rather that of biological correctness of the aligned edges [25].

To simultaneously optimize both node and edge conservation, our new method, which we call GRaphlet Edge AlignmenT (GREAT), first aims to optimally align edges between two networks, and based on the resulting edge alignment, it constructs (as we will show) a more efficient node cost function compared to state-of-the-art node similarity measures that are typically used for this purpose. That is, when we use within a given existing alignment strategy our new edge alignmentbased node cost function, we get alignments of higher quality with respect to both node and edge conservation than when we use within the same alignment strategy an existing node cost function. Thus, we improve upon methods that aim to optimize node conservation only. At the same time, GREAT is comparable or superior to MAGNA that aims to optimize edge conservation only. 


\section{Methods}

GREAT consists of four algorithmic components: 1) edge cost function, 2) edge alignment strategy, 3) node cost function, and 4) node alignment strategy. Edge cost function and edge alignment strategy are used to align well edges between two networks, similar to how existing methods align nodes between two networks based on node cost function and node alignment strategy. Then, the resulting edge alignment is used to compute a novel node cost function, according to which two nodes from different networks are similar if the nodes' adjacent edges have been aligned and with high similarity with respect to the edge cost function. Then, the resulting edge alignment-based node cost function is used within an existing node alignment strategy to produce an injective node mapping between the networks. In this way, the output of GREAT can be directly compared against alignments of the existing methods. This section details the four steps of GREAT.

\subsection{GREAT's edge cost function and edge alignment strategy}

To create pairwise edge scores, GREAT uses the notion of graphlets, as follows.

Graphlets are small induced non-isomorphic subgraphs (e.g., a triangle or a square; Figure 1) [33, 34, 35, 36, 37, 38, 39, 40]. A graphlet-based node cost function was already used for network alignment by three state-of-the-art methods: GRAAL [15], H-GRAAL [16], and MI-GRAAL [22] (also, see [3, 4, 31]). This node cost function relies on node graphlet degree vector (node-GDV) [34, which counts the number of graphlets (i.e., their topologically unique node "symmetry groups", called automorphism orbits; Figure (1) that a node touches. Then, the graphlet-based node cost function computes topological similarity between extended neighborhoods of two nodes from different networks by comparing the nodes' GDVs. Hence, this function is called node-GDV-similarity [35. Recently, we extended the notion of node-GDV into edge-GDV (Figure 1) and of nodeGDV-similarity into edge-GDV-similarity, to allow for quantifying topological similarity between extended neighborhoods of two edges rather than nodes [39]. Then, we used edge-GDV-similarity as a basis for a novel superior network clustering method [39]. Here, we use for the first time edge-GDV-similarity for network alignment, and we use it as a part of our edge cost function.

The other part of our edge cost function is a novel concept of edge graphlet degree centrality (edge-GDC), which we define as a measure the complexity of the extended network neighborhood of an edge (see below for a formal definition). We introduce edge-GDC to modify the total similarity of aligning two edges, in order to favor alignment of the densest parts of the networks. Namely, edges with a given edge-GDV-similarity and with high edge-GDC (and thus dense network neighborhoods) should be aligned before correspondingly edge-GDV-similar edges with low edge-GDC [16].

We define edge-GDC analogously to our existing definition of node-GDC [38]. For a given edge $e$, we denote the $i^{\text {th }}$ coordinate of its edge-GDV (that is, the number of times edge $e$ touches orbit i) as $e_{i}$. Then: edge-GDC $(e)=\sum_{1}^{68} w_{i} * \ln \left(e_{i}+1\right)$, where $w_{i} \in[0,1]$ is the weight of orbit $i$ that accounts for dependencies between orbits, and 68 is the total number of edge orbits in 3-5-node graphlets (there is an additional orbit for the only 2-node graphlet, i.e., an edge, but we leave out this orbit, as each edge will participate in exactly one such orbit) [35, 39].

With the notions of edge-GDV-similarity and edge-GDC introduced, we define our edge cost function (ECF), i.e., the total similarity between two edges $e$ and $f$ from different networks, as:

$$
\mathrm{ECF}=\alpha \times \text { edge-GDV-similarity }(e, f)+(1-\alpha) \times \frac{\text { edge-GDC }(e)+\text { edge-GDC }(f)}{\max \left(\text { edge-GDC }\left(G_{1}\right)\right)+\max \left(\operatorname{edge}-\mathrm{GDC}\left(G_{2}\right)\right)}
$$

where $\alpha$ is a parameter in $[0,1], G_{1}$ and $G_{2}$ are the two networks being aligned, and edge-GDC $\left(G_{i}\right)$ is the maximum GDC in network $G_{i}$. For this study, we vary $\alpha$ from 0 to 1 in increments of 0.2 . The formula is designed to normalize edge cost function to $[0,1]$ range. 
A node:

2-node
graphlet

rap

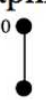

3-node graphlets

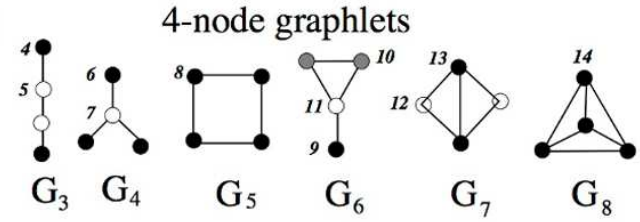

An edge:
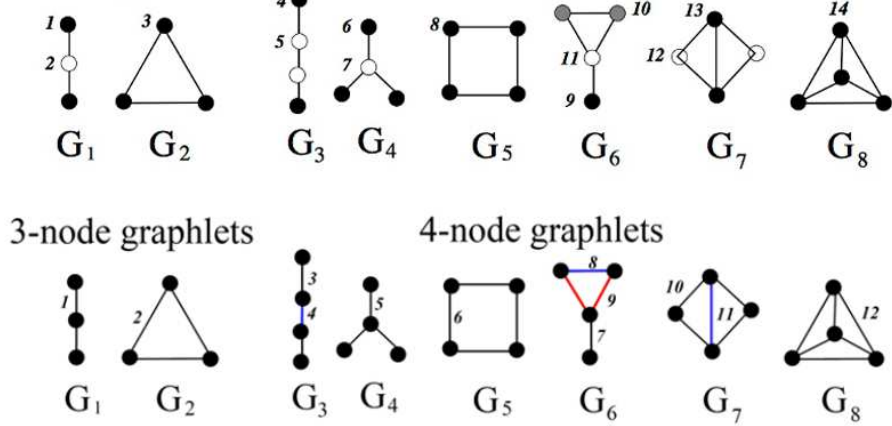

Figure 1: All automorphism orbits (i.e., topologically unique "symmetry groups") of a node (top) and an edge (bottom) in up to 4-node graphlets [34, 39. We illustrate only up to 4-node graphlets for esthetics, but all up to 5-node graphlets (with 73 node orbits and 69 edge orbits) are used within our method. The figure has been adopted and adapted from [39].

Given pairwise edge scores computed with respect to the above edge cost function, GREAT feeds these scores into an existing edge alignment strategy to produce injective edge mapping between the two networks. We use two such strategies: 1) greedy alignment strategy, which maps, one at a time, the highest-scoring edge pairs in a greedy fashion, and 2) the Hungarian algorithm for maximum weight bipartite matching, which produces optimal edge mapping with respect to our edge cost function. We use these methods as edge alignment strategies because equivalent (and thus comparable) methods have already been used in the context of network alignment as node alignment strategies, within e.g., IsoRank [18] and H-GRAAL [16], respectively. Ideally, we would have adjusted more recent and superior node alignment strategies, such as those of MI-GRAAL [22] or GHOST [23], to fit the context of our edge alignment problem. However, generalizing these node alignment strategies into analogous (and thus comparable) edge alignment strategies is non-trivial, as the current implementations of MI-GRAAL or GHOST either rely on proprietary libraries or are too complex to be extended in any way, respectively.

\subsection{GREAT's node cost function and node alignment strategy}

After generating an edge alignment, GREAT continues onto calculating pairwise node scores based on this alignment, as follows. Let $v_{1}$ be a node in graph $G_{1}$ and let $v_{2}$ be a node in graph $G_{2}$. Let $E^{\prime}$ be the set of aligned edges and let $\operatorname{sim}\left(v_{1}, v_{2}\right)$ be the similarity between $v_{1}$ and $v_{2}$. Then, GREAT's edge alignment-based node cost function $\operatorname{sim}\left(v_{1}, v_{2}\right)$ is the sum of similarities (with respect to edge cost function) over all edges in $E^{\prime}$ (Appendix Figure A.1).

After GREAT generates node cost function as above, it feeds the resulting node scores into an existing node alignment strategy to generate an injective node between the two. Here, we use three such strategies: 1) greedy alignment strategy, 2) Hungarian algorithm for maximum weight bipartite matching, and 3) MI-GRAAL's alignment strategy. Again, we would have used a more recent node alignment strategy, such as GHOST's, but the current implementation of GHOST is too complex to allow for feeding into its alignment strategy any node cost function but its own.

\subsection{Variations of GREAT}

By mixing and matching different options for GREAT's edge and node alignment strategies, as discussed above, we get a total of six different GREAT variations, i.e., aligners (Table 1). 


\begin{tabular}{|l|l|l|}
\hline GREAT variation & Edge alignment strategy & Node alignment strategy \\
\hline EGG & Greedy & Greedy \\
\hline EHG & Hungarian & Greedy \\
\hline EGH & Greedy & Hungarian \\
\hline EHH & Hungarian & Hungarian \\
\hline EGM & Greedy & MI-GRAAL \\
\hline EHM & Hungarian & MI-GRAAL \\
\hline
\end{tabular}

Table 1: Six variations of GREAT, depending on which edge and node alignment strategy it uses.

\subsection{Network data}

To test GREAT's performance, we use a popular evaluation test [15, 16, 22, 23, 28, 3, 4, 31. Namely, we focus on a high-confidence yeast PPI network with 1,004 proteins and 8,323 PPIs [41], and we produce five additional "synthetic" networks by adding noise to the yeast network. The noise is the addition to the original yeast network of $x \%$ of low-confidence PPIs from the same data set [41], where we vary $x$ from $5 \%$ to $25 \%$ in increments of $5 \%$. We align the original yeast network to each of the synthetic networks with $x \%$ noise, resulting in the total of five network pairs to be aligned. Importantly, since all network pairs have the same set of nodes, we know the true node correspondence (i.e., mapping). Thus, for each considered method, we can measure how well the method reconstructs the correspondence, along with evaluating the method's alignment quality with respect to some other measures (Section [2.5).

We note the main focus of our paper is a fair evaluation of our new GREAT method against the existing methods. If we aimed to predict new biological knowledge, we would have applied our method to additional networks, such as PPI networks of different species. However, since our main focus is method evaluation, we focus on the above network data set because: 1) the original yeast network is of high confidence and thus trustable; 2) the data encompasses different PPI types, including PPIs obtained via affinity purification followed by mass spectrometry (AP/MS), and as such is of high coverage, 3) the same data has already been actively used for evaluation of different network aligners, and 4) we know the true node mapping as well as the actual level of structural difference (corresponding to the given percentage of the low-confidence PPIs) between each pair of aligned networks, and hence, we can meaningfully interpret our alignments (where this is not the case for networks with unknown node mapping or unknown structural difference) [15, 16, 22, 23, 3, 28, 44. Ultimately, what matters for a fair evaluation is that all compared methods are tested on the same data, which is exactly what we do [4].

\subsection{Network alignment quality measures}

Let $G_{1}=\left(V_{1}, E_{1}\right)$ and $G_{2}\left(V_{2}, E_{2}\right)$ be graphs such that $V_{1} \leq V_{2}$. Let $E_{2}^{\prime}$ be the set of edges in $G_{2}$ that exist between the set of nodes in $G_{2}$ that are aligned to nodes in $G_{1}$. Then, we measure alignment quality with respect to the following well-established measures [15, 16, 22, 23, 28, 3, 4, 131.

Node correctness (NC): If $f: V_{1} \rightarrow V_{2}$ is the correct node mapping of $G_{1}$ to $G_{2}$ and $h: V_{1} \rightarrow V_{2}$

is an alignment produced by the given method, then $N C=\frac{\left|\left\{u \in V_{1}: f(u)=h(u)\right\}\right|}{\left|V_{1}\right|} \times 100 \%$ [15]. This measure can only be used on networks with known node mapping, such as our data.

Symmetric substructure score $\left(S^{3}\right)$ : Although NC captures the amount of true node mapping, it is still important to measure the amount of conserved edges. For example, if we map an $n$-node clique (complete graph) in one network to an $n$-node clique in another network, there are many possible topologically correct alignments with respect to $\mathrm{S}^{3}$, i.e., alignments that conserve all edges, but 
there is a single correct alignment with respect to NC. Plus, true node mapping is not known for most real-world networks; in such cases, $\mathrm{NC}$ can not be computed and one needs to rely on measures of edge conservation. One such measure is $\mathrm{S}^{3}$, defined as the percentage of conserved edges out of all edges in $E_{1}$ and $E_{2}^{\prime}$ combined. More formally, it is defined as follows: $S^{3}=\frac{\left|E_{1} \cap E_{2}^{\prime}\right|}{\left|E_{1}\right|+\left|E_{2}^{\prime}\right|-\left|E_{1} \cap E_{2}^{\prime}\right|} \times 100 \%$ [28]. Alternative measures of edge conservation exist, such as edge correctness [15] and induced conserved structure [23], but $\mathrm{S}^{3}$ combines the advantages of both of these measures while addressing their drawbacks, and as such, it has been shown to be the superior of the three measures [28].

The size of the largest connected common subgraph (LCCS) [15], which we use because of two alignments with similar $S^{3}$ scores, one could expose large, contiguous, and topologically complex regions of network similarity, while the other could fail to do so. Thus, in addition to counting aligned edges, it is important that the aligned edges cluster together to form large connected subgraphs rather than being isolated. Hence, a connected common subgraph (CCS) is defined as a connected subgraph (not necessarily induced) that appears in both networks [16. We measure the size of the largest CCS (LCCS) in terms of the number of nodes as well as edges. Namely, we compute the LCCS score as in our recent work [28]. First, we count $N$, the percentage of nodes from $G_{1}$ that are in the LCCS. Then, we count $E$, the percentage of edges that are in the LCCS out of all edges that could have been aligned between the nodes in the LCCS. That is, $E$ is the minimum of the number of edges in the subgraph of $G_{1}$ that is induced on the nodes from the LCCS, and the number of edges in the subgraph of $G_{2}$ that is induced on the nodes from the LCCS

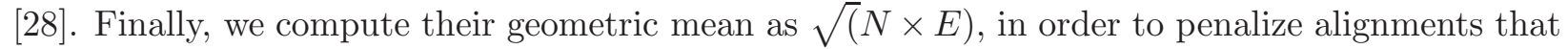
have small $N$ or small $E$. Large values of this final LCCS score are desirable.

\section{Results and discussion}

We aim to answer the following: 1) What parameter values to use within GREAT's edge cost function to optimally balance between edge-GDV-similarity and edge-GDC (Section 3.1)? 2) Does edge-based network alignment improve upon comparable traditional node-based network alignment (Section 3.2)? This is the main goal of our study, and achieving it would be sufficient to demonstrate GREAT's superiority over the traditional methods. 3) Given that we will demonstrate that edge alignment does improve over node alignment, which of the two edge alignment strategies (greedy versus optimal) to use to achieve both high accuracy and low computational complexity (Section (3.3)? That is, can we still achieve with fast greedy edge alignment similar accuracy as with slower optimal edge alignment? This has important implications for processing large real-world networks. 4) Can GREAT, our edge-based network alignment method, not only beat comparable node-based network alignment methods (question 2 above) but also the most recent and thus advanced existing network aligners (which would only further confirm GREAT's superiority) (Section 3.4)?

\subsection{Best parameter values within GREAT's edge cost function}

Recall from Section 2.1 that the $\alpha$ parameter controls the contribution of edge-GDV-similarity and edge-GDC in GREAT's edge cost function. When we test its effect on a comprehensive network set (see below), we find that overall, $\alpha$ of 1 is superior (although $\alpha$ of 0.8 performs relatively well too) (Figure 2). That is, edge-GDV-similarity is overall favored over edge-GDC. We note that this was not the case with a comparable node cost function [16], where some contribution of node centrality was desired, which is why we tested the effect of edge-GDC in GREAT's edge cost function in the first place. Henceforth, in subsequent analyses, we consider only the dominant $\alpha$ of 1 .

We have performed this analysis on synthetic (geometric and scale-free [36]) networks of different sizes (we varied the number of nodes from 500 to 1,000, and for a given node size, we varied the 
average degree from four to 12). We have aligned each such network to its noisy counterpart. Here, by noisy counterpart, we mean that in a given synthetic network, we have rewired $x \%$ of the network's edges, where $x \in\{5,12,15,20,25\}$. We have done this on the synthetic (geometric and scale-free) network data rather than on our yeast network data from Section 2.4, since we needed to test many different values of $\alpha$, and doing so on relatively large (dense) yeast networks is more time consuming. Also, we wanted to test the effect of $\alpha$ on alignment quality as a function of network size, and the yeast data does not allow for this. But henceforth, when we test actual alignment accuracy, we focus only on the yeast network data.

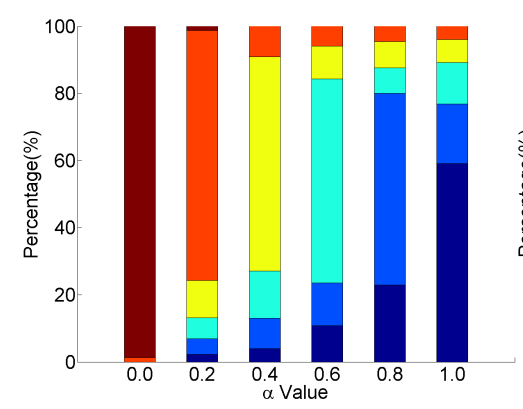

(a)

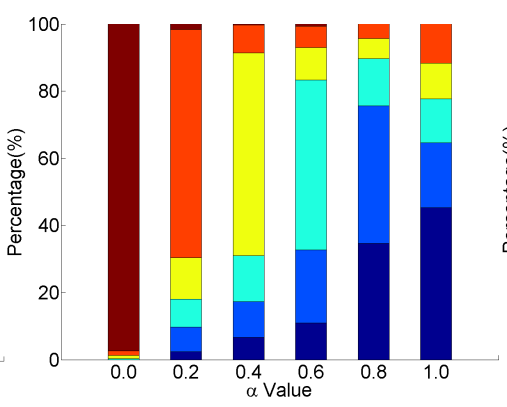

(b)

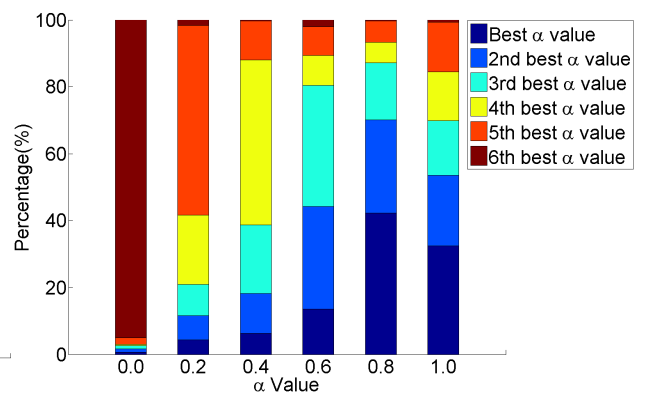

(c)

Figure 2: The ranking of the six $\alpha$ values used in GREAT's edge cost function across all variations of GREAT and all synthetic network alignments with respect to: (a) NC, (b) $\mathrm{S}^{3}$, and (c) LCCS. Recall that $\alpha=1$ corresponds to using only edge-GDV-similarity, while $\alpha=0$ corresponds to using only edge-GDC (Equation 11).

\subsection{Is edge alignment worth it compared to node alignment?}

Recall that there are different GREAT variations depending on the choices of edge and node alignment strategies (Table 1). To fairly evaluate whether edge-based network alignment improves upon node-based network alignment, we benchmark a given variation of GREAT against the comparable node-based network alignment method. That is, recall that a given version of GREAT uses edge-GDV-similarity-based edge cost function (corresponding to $\alpha$ of 1), a given (greedy or optimal) edge alignment strategy, the edge-alignment-based node cost function, and a given (greedy, optimal (Hungarian), or MI-GRAAL's) node alignment strategy. Given this, we produce the corresponding node-based network alignment method as follows: it uses node-GDV-similarity as its node cost function and the same node alignment strategy as the given version of GREAT. This way, because edge-GDV-similarity and node-GDV-similarity are as fairly comparable as possible measures of topological similarity of edges and nodes, respectively, and because we are using the same node alignment strategy in both GREAT and the corresponding node alignment-based method, any difference that we observe between the two methods will be a direct consequence of edge-based network alignment compared to node-based alignment.

Consequently, we denote by NG the node-based alignment method that uses the greedy node alignment strategy and by NH the node-based alignment method that uses the Hungarian node alignment strategy. The node-based alignment method that uses MI-GRAAL's node alignment strategy is MI-GRAAL itself. Then, we compare each of EGG and EHG to NG, each of EGH and EHH to NH, and each of EGM and EHM to MI-GRAAL (Table 1).

Overall, across all network alignment measures, we find that edge alignment is superior to node alignment (Figure 3 and Appendix Figure A.2). That is, we demonstrate that edge-based network alignment outperforms node-based network alignment when using comparable cost functions and alignment strategies, which is the main contribution of our study. 


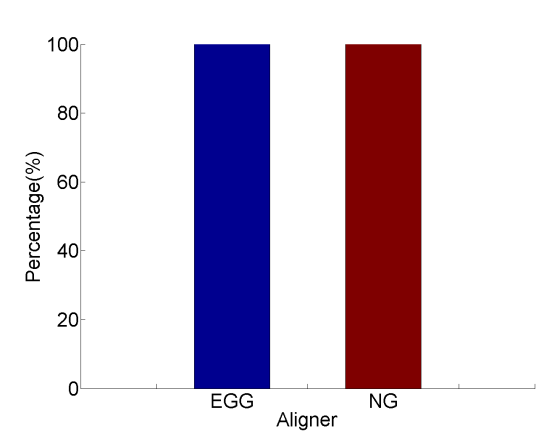

(a)

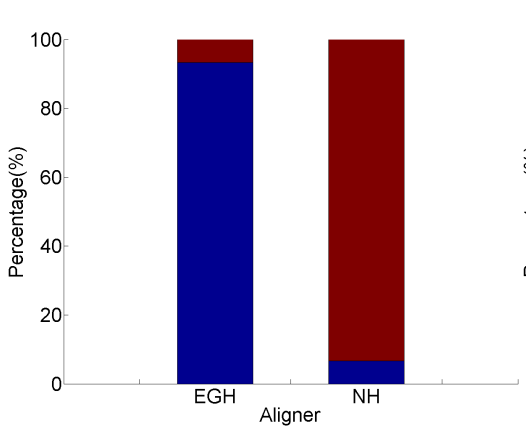

(b)

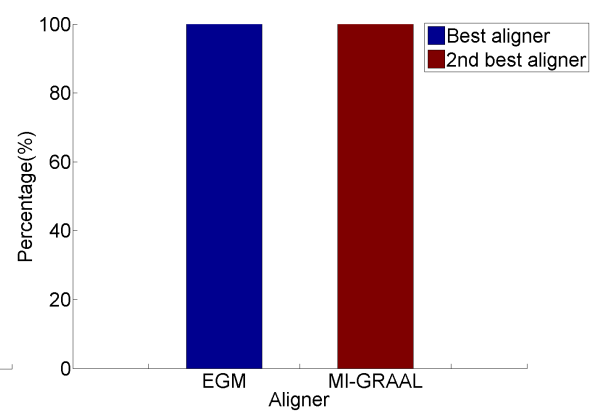

(c)

Figure 3: The ranking of a given edge-based network aligner and the corresponding node-based aligner across all alignments with respect to all alignment quality measures for: (a) greedy, (b) Hungarian, and (c) MI-GRAAL's node alignment strategy. Note that these results are for greedy edge alignment strategy only, but almost identical results are obtained for optimal edge alignment strategy, i.e., when comparing EHG with NG, EHH with NH, and EHM with MI-GRAAL.

\subsection{Speed of greedy edge alignment versus accuracy of optimal edge alignment}

Overall, aligning edges optimally with the Hungarian strategy is expected to outperform (in terms of accuracy) aligning edges with the greedy strategy. However, Hungarian method is much slower than the greedy method, with complexity of $\mathcal{O}\left(x^{3}\right)$ for the former and $\mathcal{O}(x)$ for the latter, where $x$ is the number of elements (in this case, edges) to be aligned. So, the question is to what extent optimal edge alignment improves compared to greedy alignment, and whether this increase in accuracy is worth the drastic increase in running time.

To fairly evaluate this, we compare EGG to EHG, EGH to EHH, to EGM and EHM. In Table2, we show representative running times and alignment accuracy scores (in terms of $S^{3}$ measure) of EGH and EHH, and in Figure 4 we show systematic results for all GREAT versions while taking into account all measures of alignment quality. As illustrated, aligning edges with the Hungarian method yields to only 0\%-14\% increase (depending on the network data) in accuracy compared aligning edges greedily, but it leads to extremely large 5,271\%-13,407\% increase in running time (Table 2). Further, in the systematic analysis, we find that in $47 \%-73 \%$ of all cases (depending on node alignment strategy) accuracy of greedy edge alignment is within $5 \%$ of accuracy of optimal edge alignment (Figure 4), and the percentages are even higher for being within 10\% accuracy (Appendix Figure A.3). Thus, we believe that the huge increase in computational complexity of optimal edge alignment does not justify incremental increase in its accuracy. Henceforth, especially for large networks, we suggest aligning edges greedily. (Given the resulting edge alignment-based node cost function, one still might want to align nodes optimally.)

\subsection{GREAT versus the most recent and advanced methods}

Here, we compare GREAT (the best of all variations) against the following recent powerful aligners: MI-GRAAL [22], GHOST [23], NETAL [24], and MAGNA [28]. We use default parameters (suggested in the original publications) for all methods. We also tried SPINAL [32, but it did not return injective node mappings on our network data as the other methods and was thus excluded.

We find that GREAT is overall the best aligner across all alignment quality measures (Figure 5 (a) and Appendix Figure A.4). This is especially true in terms of NC, which is the ultimate measure of alignment accuracy - GREAT is always the best of all methods (Figure 5 (b)). In terms of $S^{3}$, only MAGNA is the best ranked in more cases than GREAT (Figure 5 (c)). However, 


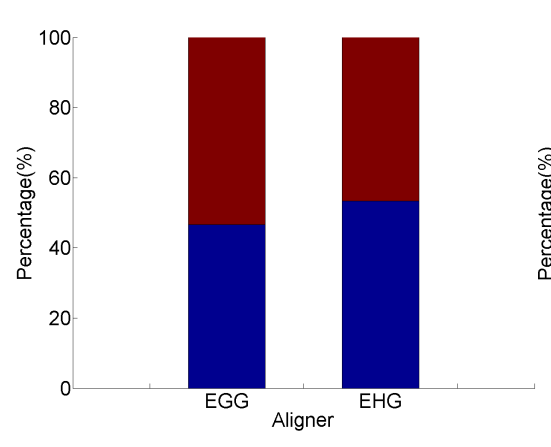

(a)

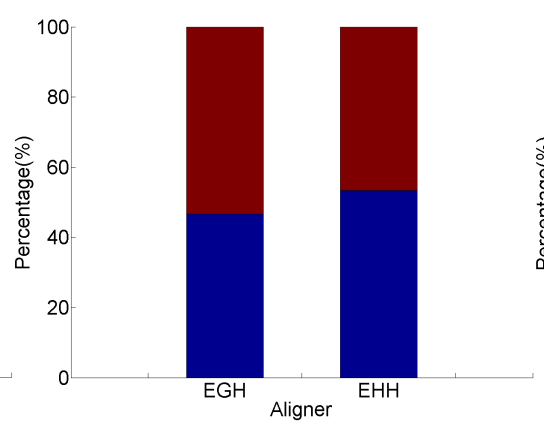

(b)

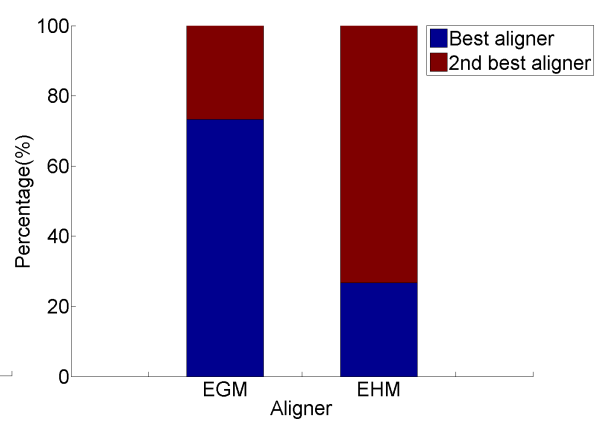

(c)

Figure 4: The ranking of a given greedy edge aligner and the corresponding optimal edge aligner across all alignments with respect to all alignment quality measures for: (a) greedy, (b) Hungarian, and (c) MI-GRAAL's node alignment strategy, for "within 5\% accuracy". By this, we mean that the greedy edge aligner's score is within $5 \%$ of the optimal edge aligner's score.

\begin{tabular}{|c|c|c|c|c|c|c|}
\hline Alignme & \multicolumn{2}{|c|}{ EGH } & \multicolumn{2}{|l|}{$\mathrm{EHH}$} & \multicolumn{2}{|c|}{ Percentage Increase } \\
\hline & Time & $\mathrm{S}^{3}$ & Time & $\mathrm{S}^{3}$ & Time & $\mathrm{S}^{3}$ \\
\hline Yeast-Yeast5\% & $3 \mathrm{~h} 49 \mathrm{~m} 24 \mathrm{~s}$ & $91.97 \%$ & $220 \mathrm{~h} 20 \mathrm{~m} \mathrm{03s}$ & $92.33 \%$ & $5,271 \%$ & $0.34 \%$ \\
\hline Yeast-Yeast10\% & $4 \mathrm{~h} 16 \mathrm{~m} 08 \mathrm{~s}$ & $71.91 \%$ & $432 \mathrm{~h} 29 \mathrm{~m} 14 \mathrm{~s}$ & $74.05 \%$ & $10,031 \%$ & $2.98 \%$ \\
\hline Yeast-Yeast15\% & $5 \mathrm{~h} 00 \mathrm{~m} 38 \mathrm{~s}$ & $54.70 \%$ & $570 \mathrm{~h} 48 \mathrm{~m} 22 \mathrm{~s}$ & $60.13 \%$ & $11,292 \%$ & $9.93 \%$ \\
\hline Yeast-Yeast20\% & $5 \mathrm{~h} 07 \mathrm{~m} 06 \mathrm{~s}$ & $45.04 \%$ & $691 \mathrm{~h} 18 \mathrm{~m} \mathrm{40s}$ & $46.77 \%$ & $13,407 \%$ & $3.84 \%$ \\
\hline Yeast-Yeast25\% & $5 \mathrm{~h} 37 \mathrm{~m} 59 \mathrm{~s}$ & $34.45 \%$ & $755 \mathrm{~h} 36 \mathrm{~m} \mathrm{06s}$ & $39.25 \%$ & $13,314 \%$ & $13.93 \%$ \\
\hline
\end{tabular}

Table 2: Computational complexity versus accuracy of greedy versus optimal edge alignment. We show the amount of CPU time it took GREAT variations EGH and EHH to generate an alignment and $S^{3}$ score of the resulting alignment, for each pair of yeast networks. We measure the increase in either running time or accuracy of EHH (i.e., optimal edge alignment) over EGH (i.e., greedy edge alignment) as the difference of the result of EHH and the result of EGH, divided by the result of EGH. All alignments were ran on the same server with $162.3 \mathrm{GHz}$ processors and 24GB of RAM.

this is not surprising, as MAGNA directly optimizes $\mathrm{S}^{3}$ during alignment construction and is thus expected to dominate the other methods with respect to this measure. Nonetheless, GREAT still outperforms MAGNA is $40 \%$ of the cases with respect to $\mathrm{S}^{3}$. Finally, in terms of LCCS, GREAT is again superior to almost all methods, including MAGNA (Figure 5 (d)). Exceptions are GHOST and NETAL, but GREAT still performs comparably to these methods, in the sense that all three methods rank as the best or the second best in equal number (60\%) of all cases.

Thus, we do not only demonstrate that GREAT is superior to fairly comparable node-based network alignment methods, which differ from GREAT in a single aspect (edge versus node alignment), but also, it is superior to the recent state-of-the-art network alignments, which differ from GREAT in more than one aspect. As such, incorporating into the design of GREAT the recent methods' algorithmic ideas could potentially improve GREAT's performance even further.

\section{Conclusion}

We have presented GREAT, our novel alignment method that aims to maximize both node and edge conservation, that does so by first aligning edges well in order to then align nodes well based on their adjacent edges being aligned well, and that in the process favors similar conserved edges over dissimilar conserved edges. We have demonstrated that GREAT, the edge-based network align- 


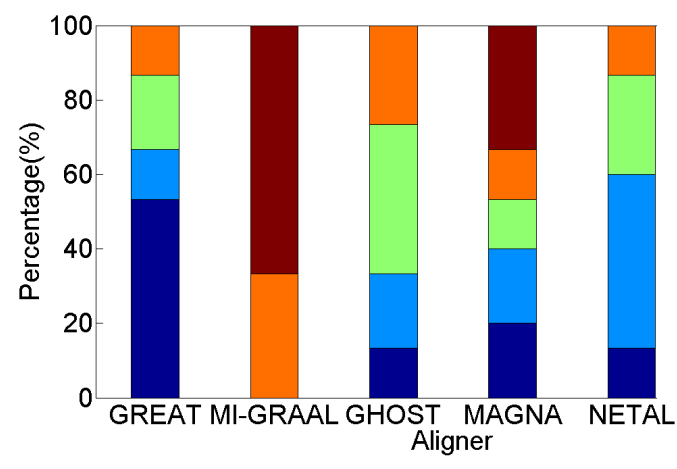

(a)

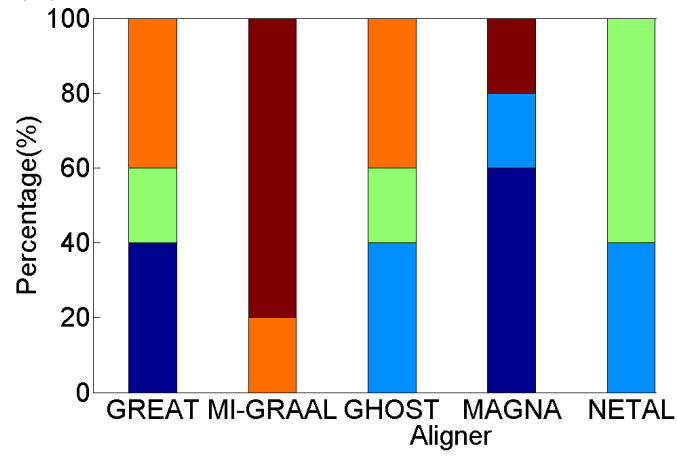

(c)

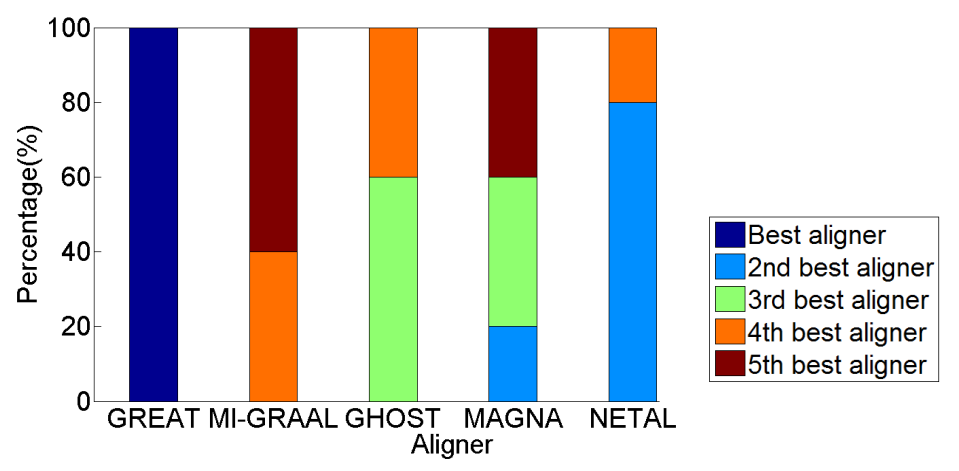

(b)

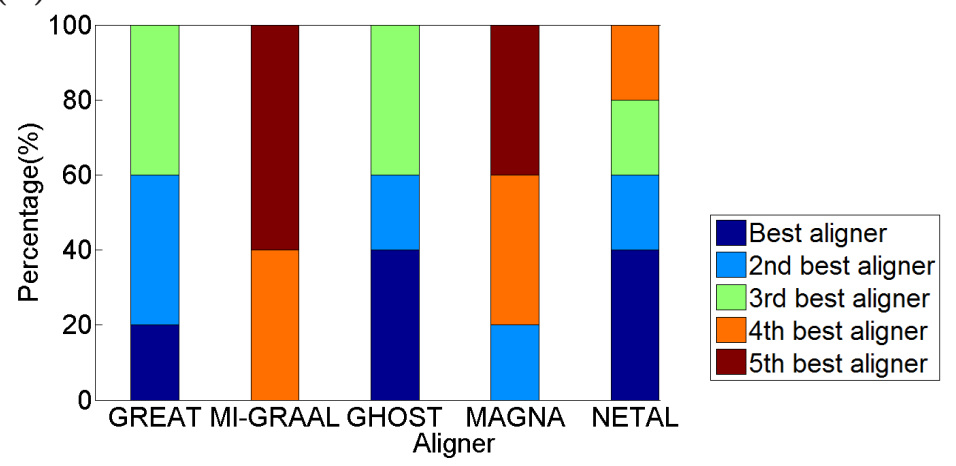

(d)

Figure 5: The ranking of GREAT (the best of all variations) and very recent and thus advanced existing network aligners over all alignments with respect to: (a) all alignment quality measures combined, (b) NC, (c) $\mathrm{S}^{3}$, and (d) LCCS.

ment method, improves upon comparable node-based network alignment methods, confirming our hypothesis that aligning edges prior to aligning nodes would improve alignment quality compared to aligning nodes only. In other words, we have demonstrated superiority of GREAT over methods that aim to maximize node conservation only, such as MI-GRAAL and GHOST. At the same time, we have demonstrated superiority of GREAT over a recent approach that aims to optimize edge conservation only and that treats each edge the same, namely MAGNA. Finally, we have shown that GREAT overall outperforms an additional recent state-of-the-art approach, namely NETAL.

Thus, GREAT (and its modified version that would also account for functional, e.g., protein sequence, similarities between nodes in addition to their topological similarities) has important implications for real-world applications of network alignment to biological networks of different species, as well as to networks in other domains, such as social networks or natural language processing. For example, in computationally biology, GREAT can be used to transfer aging-related knowledge from well-annotated model species to poorly-annotated human, thus deepening our current knowledge about human aging [3, 4, 5]. Or, it could have implications on user privacy in online social networks, as network alignment can be used to de-anonymize such network data [2].

\section{Acknowledgements}

We thank Dr. Horst Bunke for useful discussions regarding GREAT, and Dr. Seyed Shahriar Arab for his assistance with running NETAL. This work was supported by the National Science Foundation CCF-1319469 and EAGER CCF-1243295 grants. 


\section{References}

[1] J. Li, J. Tang, Y. Li, and Q. Luo. RiMOM: A dynamic multistrategy ontology alignment framework. Knowledge and Data Engineering, IEEE Transactions on, 21(8):1218-1232, 2009.

[2] A. Narayanan, E. Shi, and B. IP Rubinstein. Link prediction by de-anonymization: How we won the Kaggle social network challenge. In Neural Networks (IJCNN), The 2011 International Joint Conference on, pages 1825-1834. IEEE, 2011.

[3] T. Milenković, H. Zhao, and F. E. Faisal. Global network alignment in the context of aging. In Proceedings of the International Conference on Bioinformatics, Computational Biology and Biomedical Informatics, BCB'13, pages 23:23-23:32, 2013.

[4] F. Faisal, H. Zhao, and T. Milenkovic. Global network alignment in the context of aging. Computational Biology and Bioinformatics, IEEE/ACM Transactions on Computational Biology and Bioinformatics, PP(99), 2014.

[5] F. E. Faisal and T. Milenković. Dynamic networks reveal key players in aging. Bioinformatics, 30(12):1721-1729, 2014.

[6] B. P. Kelley, V. Yuan, F. Lewitter, R. Sharan, B. R. Stockwell, and T. Ideker. PathBLAST: a tool for alignment of protein interaction networks. Nucleic Acids Research, 32:83-88, 2004.

[7] R. Sharan, S. Suthram, R.M. Kelley, T. Kuhn, S. McCuine, P. Uetz, T. Sittler, R.M. Karp, and T. Ideker. Conserved patterns of protein interaction in multiple species. Proceedings of the National Academy of Sciences, 102(6):1974-1979, 2005.

[8] J. Flannick, A. Novak, S.S. Balaji, H.M. Harley, and S. Batzglou. Graemlin general and robust alignment of multiple large interaction networks. Genome Research, 16(9):1169-1181, 2006.

[9] M. Koyuturk, Y. Kim, U. Topkara, S. Subramaniam, W. Szpankowski, and A. Grama. Pairwise alignment of protein interaction networks. Journal of Computational Biology, 13(2), 2006.

[10] J. Berg and M. Lassig. Local graph alignment and motif search in biological networks. Proceedings of the National Academy of Sciences, 101(41):14689-14694, 2004.

[11] Z. Liang, M. Xu, M. Teng, and L. Niu. NetAlign: A web-based tool for comparison of protein interaction networks. Bioinformatics, 22(17):2175-2177, 2006.

[12] J. Berg and M. Lassig. Cross-species analysis of biological networks by Bayesian alignment. Proceedings of the National Academy of Sciences, 103(29):10967-10972, 2006.

[13] M. Mina and P. Hiram Guzzi. Improving the robustness of local network alignment: design and extensive assessment of a markov clustering-based approach. IEEE/ACM Transactions on Computational Biology and Bioinformatics, 99(PrePrints):1, 2014.

[14] G. Ciriello, M. Mina, P. H. Guzzi, M. Cannataro, and C. Guerra. AlignNemo: A Local Network Alignment Method to Integrate Homology and Topology. PLOS ONE, 7(6), 2012.

[15] O. Kuchaiev, T. Milenković, V. Memišević, W. Hayes, and N. Pržulj. Topological network alignment uncovers biological function and phylogeny. Journal of The Royal Society Interface, 7(50):1341-1354, 2010.

[16] T. Milenković, W.L. Ng, W. Hayes, and N. Pržulj. Optimal network alignment with graphlet degree vectors. Cancer Informatics, 9:121-137, 2010.

[17] C. Liao, K. Lu, M. Baym, R. Singh, and B. Berger. IsoRankN: Spectral methods for global alignment of multiple protein networks. Bioinformatics, 25(12):i253-258, 2009.

[18] R. Singh, J. Xu, and B. Berger. Pairwise global alignment of protein interaction networks by matching neighborhood topology. In Research in computational molecular biology, pages 16-31. Springer, 2007. 
[19] J. Flannick, A. Novak, C. B. Do, B. S. Srinivasan, and S. Batzoglou. Automatic parameter learning for multiple network alignment. In Proceedings of the 12th annual international conference on Research in computational molecular biology, pages 214-231, 2008.

[20] R. Singh, J. Xu, and B. Berger. Global alignment of multiple protein interaction networks. In Proceedings of Pacific Symposium on Biocomputing 13, pages 303-314, 2008.

[21] M. Zaslavskiy, F. Bach, and J. P. Vert. Global alignment of protein-protein interaction networks by graph matching methods. Bioinformatics, 25(12):i259-i267, 2009.

[22] O. Kuchaiev and N. Pržulj. Integrative network alignment reveals large regions of global network similarity in yeast and human. Bioinformatics, 27(10):1390-1396, 2011.

[23] R. Patro and C. Kingsford. Global network alignment using multiscale spectral signatures. Bioinformatics, 28(23):3105-3114, 2012.

[24] B. Neyshabur, A. Khadem, S. Hashemifar, and S. Shahriar Arab. NETAL: a new graph-based method for global alignment of protein-protein interaction networks. Bioinformatics, 29(13):1654-1662, 2013.

[25] X. Guo and A.J. Hartemink. Domain-oriented edge-based alignment of protein interaction networks. Bioinformatics, 25(12):i240-1246, 2009.

[26] G.W. Klau. A new graph-based method for pairwise global network alignment. BMC Bioinformatics, 10(Suppl 1):S59, 2009.

[27] M. El-Kebir, J. Heringa, and G. W Klau. Lagrangian relaxation applied to sparse global network alignment. In Pattern Recognition in Bioinformatics, pages 225-236. 2011.

[28] Vikram Saraph and Tijana Milenkovi. Magna: Maximizing accuracy in global network alignment. Bioinformatics, 30(20):2931-2940, 2014.

[29] C. Clark and J. Kalita. A comparison of algorithms for the pairwise alignment of biological networks. Bioinformatics, DOI: 10.1093/bioinformatics/btu307, 2014.

[30] D.B. West. Introduction to Graph Theory. Prentice Hall, Upper Saddle River,NJ, $2^{\text {nd }}$ edition, 2001.

[31] J. Crawford, Y. Sun, and T. Milenković. Fair evaluation of global network aligners. arXiv:1407.4824, 2014.

[32] Ahmet E Aladağ and Cesim Erten. Spinal: scalable protein interaction network alignment. Bioinformatics, 29(7):917-924, 2013.

[33] N. Pržulj, D. G. Corneil, and I. Jurisica. Modeling interactome: Scale-free or geometric? Bioinformatics, 20(18):3508-3515, 2004.

[34] N. Pržulj. Biological network comparison using graphlet degree distribution. Bioinformatics, 23:e177e183, 2007.

[35] T. Milenković and N. Pržulj. Uncovering biological network function via graphlet degree signatures. Cancer Informatics, 6:257-273, 2008.

[36] T. Milenković, J. Lai, and N. Pržulj. GraphCrunch: a tool for large network analyses. BMC Bioinformatics, 9(70), 2008.

[37] T. Milenković, V. Memisević, A. K. Ganesan, and N. Pržulj. Systems-level cancer gene identification from protein interaction network topology applied to melanogenesis-related interaction networks. Journal of the Royal Society Interface, 7(44):423-437, 2010.

[38] T. Milenković, V. Memišević, A. Bonato, and N. Pržulj. Dominating biological networks. PLOS ONE, 6(8):e23016, 2011.

[39] R.W. Solava, R.P. Michaels, and T. Milenković. Graphlet-based edge clustering reveals pathogeninteracting proteins. Bioinformatics, 18(28):i480-i486, 2012. 
[40] Y. Hulovatyy, R.W. Solava, and T. Milenković. Revealing missing parts of the interactome via link prediction. PLOS ONE, 9(3):e90073, 2014.

[41] S.R. Collins, P. Kemmeren, X.C. Zhao, J.F. Greenblatt, F. Spencer, F.C.P. Holstege, J.S. Weissman, and N.J. Krogan. Toward a comprehensive atlas of the phyisical interactome of Saccharomyces cerevisiae. Molecular Cell Proteomics, 6(3):439-450, 2007. 


\section{Appendix for:}

\section{GREAT: GRaphlet Edge-based network AlignmenT}

\section{Joseph Crawford and Tijana Milenković*}

Department of Computer Science and Engineering, Interdisciplinary Center for Network Science and Applications, and ECK Institute for Global Health, University of Notre Dame

${ }^{*}$ Corresponding Author (E-mail: tmilenko@nd.edu)
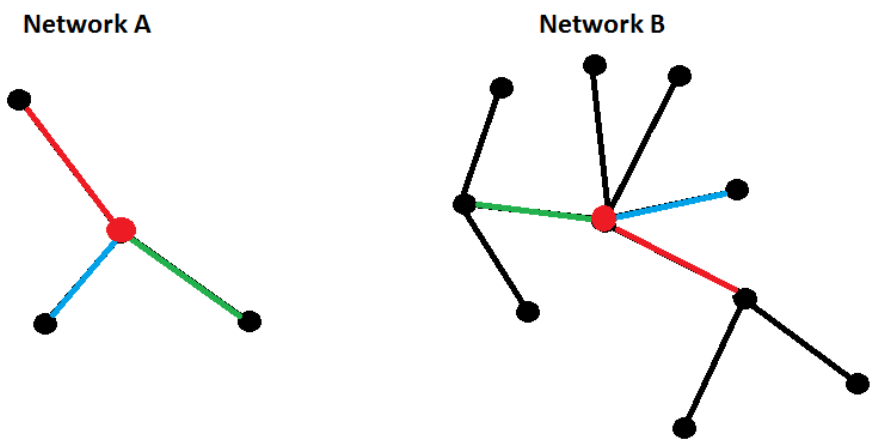

Figure A.1: Illustration of GREAT's edge alignment-based node cost function. To measure similarity between two red nodes in networks A and B, GREAT first identifies all edges adjacent to the red nodes that are aligned to each other, along with their similarity scores with respect to edge cost function. In this case, let us assume that red edge in $\mathrm{A}$ is aligned to red edge in $\mathrm{B}$ with score of 0.9 , blue edge in $\mathrm{A}$ is aligned to blue edge in $\mathrm{B}$ with score of 0.8 , and green edge in $\mathrm{A}$ is aligned to green edge in $\mathrm{B}$ with score of 0.7, while all black edges in the larger of the two networks, i.e., network B, are unaligned. Then, GREAT's edge alignment-based node cost function is the sum of similarities (with respect to edge cost function) over all aligned edges. 


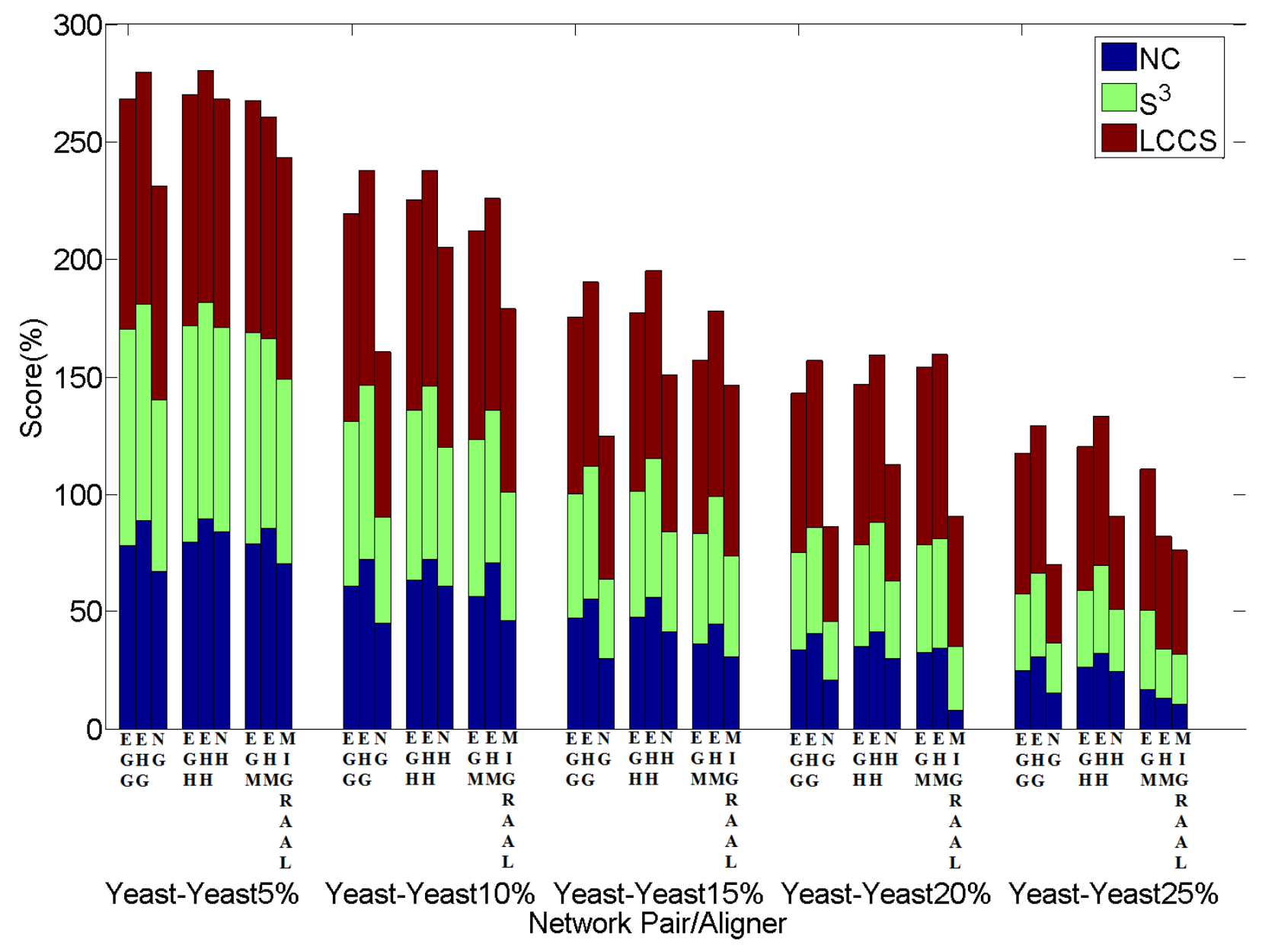

Figure A.2: Alignment quality results of all six variations of GREAT edge-based network alignment method and the corresponding three node-based network alignment methods, for each of the five network pairs and with respect to each of the three alignment quality measures.

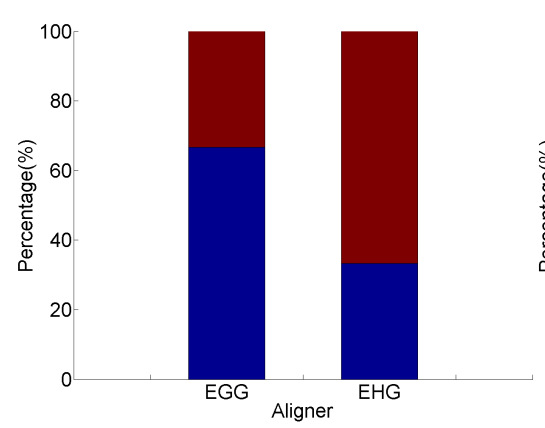

(a)

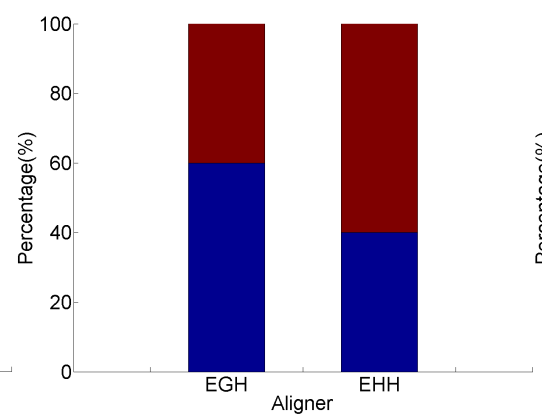

(b)

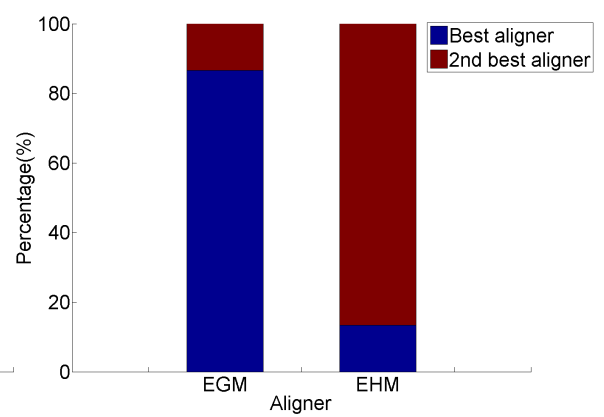

(c)

Figure A.3: The ranking of a given greedy edge aligner and the corresponding optimal edge aligner across all alignments with respect to all alignment quality measures for: (a) greedy, (b) Hungarian, and (c) MI-GRAAL's node alignment strategy, for "within 10\% accuracy". By this, we mean that the greedy edge aligner's score is within $10 \%$ of the optimal edge aligner's score. 


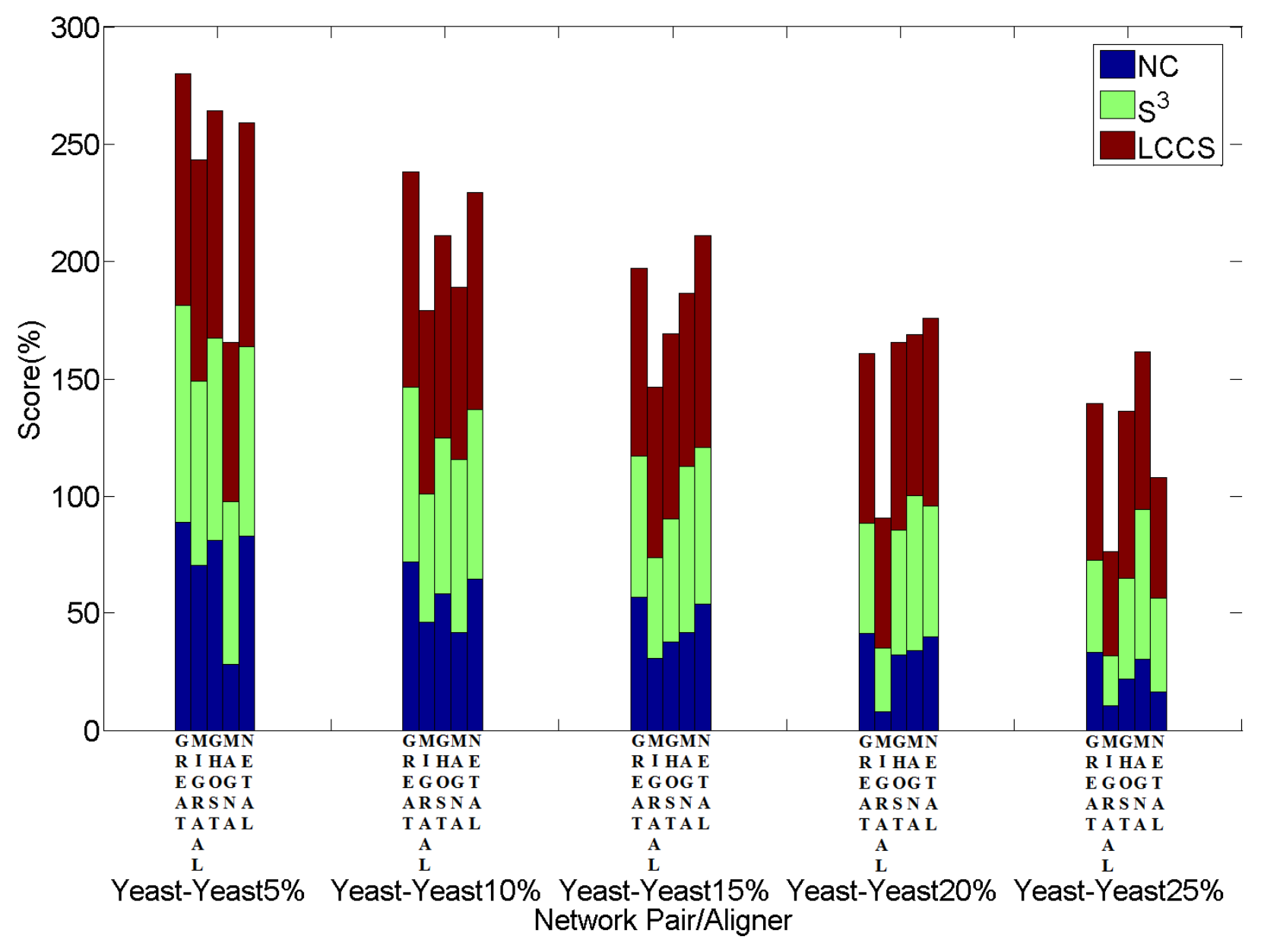

Figure A.4: Alignment quality results of GREAT (the best of all variations) and four very recent and thus advanced existing network aligners, for each of the five network pairs and with respect to each alignment quality measure. 